\title{
Dependence of the Omori-Utsu law parameters on main shock magnitude: Observations and modeling
}

\author{
S. Hainzl ${ }^{1}$ and D. Marsan ${ }^{2}$ \\ Received 9 November 2007; revised 4 June 2008; accepted 24 July 2008; published 14 October 2008.
}

[1] We examine the dependence on main shock magnitude $m$ of the $p$ and $\chi$ parameters appearing in Omori-Utsu formula $\lambda(t, m)=\chi \times(t+c)^{-p}$ relating the rate of aftershocks $\lambda$ at time $t$ after a main shock. Observations point out to a significant increase of $p$ with $m$, along with a scaling relationship of the form $\chi \sim 10^{\alpha m}$. Here we show that these observations can be explained within the framework of the rate-and-state friction model when accounting for realistic levels of coseismic stress heterogeneity on the main fault. We constrain the model parameters in order to recover the trends observed in previous and new analyses of aftershock sequences. The expected ratio of the coseismic stress drop standard deviation to its mean is found to be of the order of a few units for large $(m=7)$ earthquakes, resulting in a very rough stress field at the small scale, while it is much smoother at small magnitudes (ratio $\simeq 0.1$ at $m=2$ ). Finally, the influence of afterslip on parameters $p$ and $\chi$ is studied to highlight the fact that it can significantly perturb the $p(m)$ and $\chi(m)$ relations obtained with the initial afterslip-free model.

Citation: Hainzl, S., and D. Marsan (2008), Dependence of the Omori-Utsu law parameters on main shock magnitude: Observations and modeling, J. Geophys. Res., 113, B10309, doi:10.1029/2007JB005492.

\section{Introduction}

[2] Almost all larger earthquakes are found to trigger aftershocks with a temporal decaying probability. In particular, the occurrence rate of aftershocks $\lambda$ can be well described by the modified Omori-Utsu law

$$
\lambda(t, m)=\chi(t+c)^{-p}
$$

where $t$ indicates the elapsed time since the main shock (see Utsu et al. [1995] for a review). The $c$ value is a constant typically much less than 1 day, and in most cases is related to changes in detection level of the operating seismic network. Recent attempts at finding a $c$ value of physical rather than instrumental origin have proposed that it could be of the order of one to several minutes [Kagan and Houston, 2005; Peng et al., 2006, 2007; Enescu et al., 2007], although there is no clear consensus on how the Omori-Utsu law actually breaks down below this cutoff. The $p$ value is in the range $0.8-1.2$ in most cases [Utsu et al., 1995]. While alternative models for describing the aftershock decaying rate have been proposed [Kisslinger, 1993; Gross and Kisslinger, 1994; Narteau et al., 2002], the Omori-Utsu law generally provides a very good fit to the data and is an ubiquitous feature in seismicity dynamics.

[3] We here analyze how parameters $p$ and $\chi$ change with the magnitude $m$ of the main shock. A wealth of recent

\footnotetext{
${ }^{1}$ GFZ German Research Centre for Geosciences, Potsdam, Germany.

${ }^{2}$ Laboratoire de Geophysique Interne et Tectonophysique, Universite de Savoie, CNRS, Le Bourget du Lac, France.

Copyright 2008 by the American Geophysical Union. 0148-0227/08/2007JB005492\$09.00
}

studies have addressed the dependence of $\chi$ (mostly) with $m$, generally showing that $\chi \sim 10^{\alpha m}$. The value of parameter $\alpha$ is however variable from one study to the other, mainly because of different assumptions regarding to the definition of what main shocks and aftershocks are. Also, a significant increase of $p$ with $m$, which was not recognized before, has been recently observed by Ouillon and Sornette [2005], which they explain by a multifractal model of stress interactions [Sornette and Ouillon, 2005].

[4] The goal of this paper is to show that these observations are consistent with a model based on rate-and-state friction [Dieterich, 1994], with a spatially heterogeneous coseismic stress change at the length scale of earthquake nucleation. In section 2 , we recall results of past analyses on the magnitude dependence of $\chi$ and $p$, and test these results by new such analyses, probing different ways of selecting main shocks and aftershocks. In section 3, we detail our model, and explore its parameter space so to provide constraints on what values of these parameters can reproduce the observations. Finally, in section 4, we study how the addition of afterslip can influence the $p$ and $\chi$ values, still exploiting the rate-and-state model with coseismic stress heterogeneity.

\section{Observations}

[5] There is good evidence that the productivity $\chi$ grows exponentially with $m$, i.e., following a $\chi \sim 10^{\alpha m}$ relation. However, the exact value of $\alpha$ varies substantially between studies: Helmstetter [2003] obtained that $0.7<\alpha<0.9$ for southern California, while Felzer et al. [2004] and Helmstetter et al. [2005] found $\alpha=1$ and $\alpha=1.05 \pm 0.05$, respectively, for the same region. Using space-time ETAS 


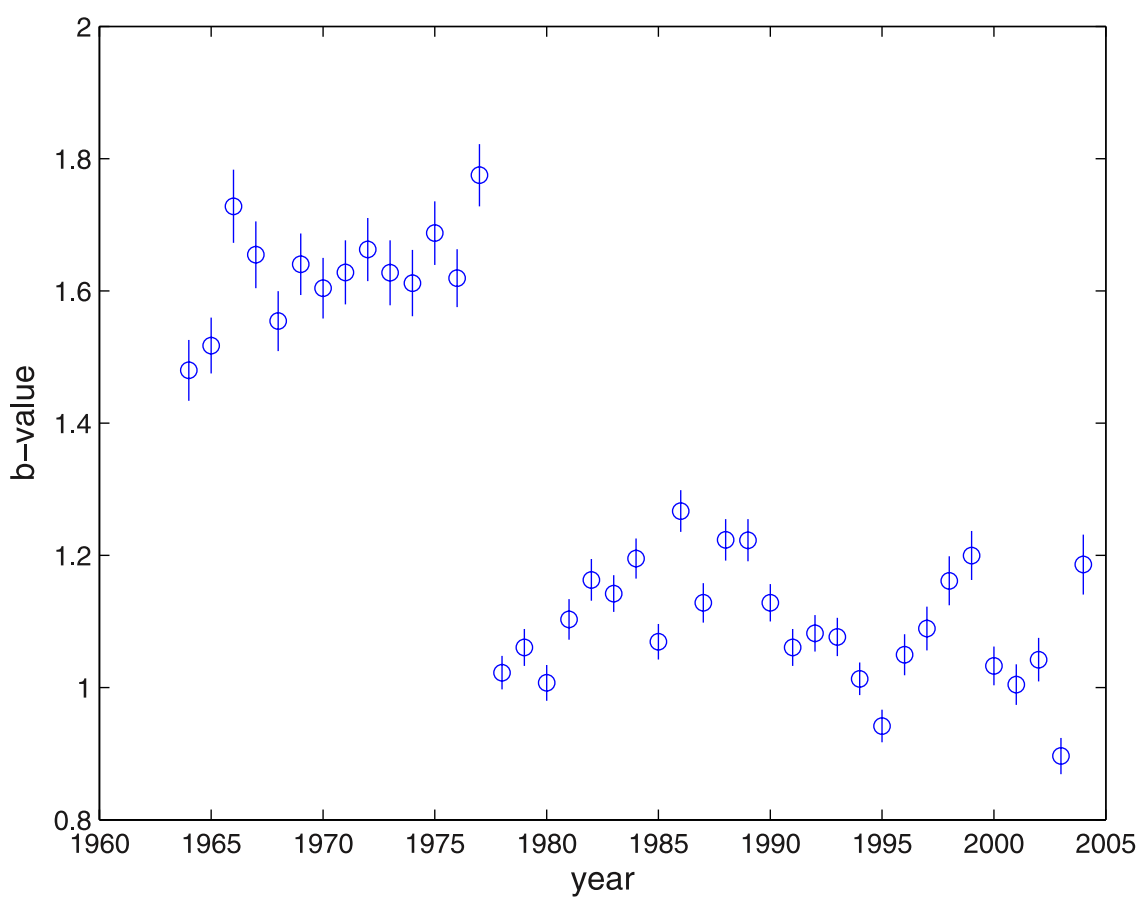

Figure 1. The $b$ value estimated for each year individually in the ISC catalog. A clear change in $b$ value is observed in 1978, when Ms magnitudes started to be reported.

models and inverting for the model parameters, Zhuang et al. [2004] found that $\alpha \simeq 0.6$ for Japan (1926-1999 $m \geq 4.2$ earthquakes), Zhuang et al. [2005] found $\alpha=0.7 \pm$ 0.05 for Taiwan (1987-2000 $m \geq 5.3$ earthquakes), while Console et al. [2003] obtained $\alpha=0.42$ for Italy (1987$2000 m \geq 2$ earthquakes).

[6] Variations in the estimate of $\alpha$ can be due to differences in the seismogenic properties of the regions analyzed, but also to the different procedures used to select which earthquakes are main shocks and which others are aftershocks. This selection is generally performed using spacetime windows that define the "aftershock domain" of a main shock [cf. Molchan and Dmitrieva, 1992]. Such methods rely on sets of parameters, that are largely arbitrary. The alternative approach of fitting ETAS model parameters to the data is computationally much more involved, and remains clearly model-dependent. Recently, a new probabilistic method (model-independent stochastic declustering, MISD) for selecting main shocks and aftershocks, that does not rely on any particular model nor specific parameterization, has been proposed [Marsan and Lengliné, 2008]. This approach, based on the premises that seismicity dynamics result from a linear cascade of earthquake triggering, permits to distinguish between directly and indirectly triggered aftershocks. Applying this method to southern California data, Marsan and Lengliné [2008] found that the $\chi \sim 10^{\alpha m}$ is indeed a good representation of the data, with an $\alpha$ parameter equal to 0.6 for directly triggered aftershocks, while $\alpha=0.66$ for all (i.e., direct and indirect) aftershocks, which is what the space-time window methods measure. In the context of the ETAS model, a single $\alpha$ parameter characterize both the direct and the overall aftershock populations. However, $\alpha$ values inverted by cascading models with an isotropic spatial kernel are likely to underestimate the real value as recently demonstrated for the case of the space-time ETAS model [Hainzl et al., 2008]. The reason is that real aftershock clusters are usually anisotropically distributed in space due to the spatial extension of main shock ruptures. Indeed, relaxing the isotropy assumption, Marsan and Lengliné [2008] found $\alpha=0.86$ ("bare" value for directly triggered events) and $\alpha=0.73$ ("dressed" value for directly and indirectly triggered events), instead of $\alpha=0.60$ and 0.66 , respectively, when assuming isotropy.

[7] To further study the $p$ and $\chi$ dependence on $m$, we here analyze the global earthquake catalog provided by the ISC, focusing on the 1978-2005 period and $m \geq 5.5$ earthquakes. The starting date of 1 January 1978 is constrained by the fact that surface wave magnitudes $m_{s}$ only start to be reported at that date. We kept the maximum magnitude (whatever its type) to characterize the size of an earthquake. This choice is purely empirical, and was motivated by the requirement that the frequency-magnitude curve follows an exponential Gutenberg-Richter law. Indeed, no deviation to the Gutenberg-Richter law above magnitude 5.5 is found when examining the global seismicity, and when analyzing each year individually. Also, as shown in Figure 1, the $b$ value remains stable over the years, indicating that there is no statistically significant change in magnitude reporting in the 1978-2005 period.

[8] We select main shocks and aftershocks using several different published selection rules, for comparison purposes. An earthquake is characterized by its time of occurrence $t$, its location $x$, and its magnitude $m$, which we use to define its rupture length as $L=10^{0.45 \times(m-6)} \times 10 \mathrm{~km}$ consistent with the analysis of Wells and Coppersmith [1994] (with a minimum $L=10 \mathrm{~km}$, hence for all earthquakes with $5.5 \leq$ $m \leq 6$, to account for location error). Namely, we use the following: 

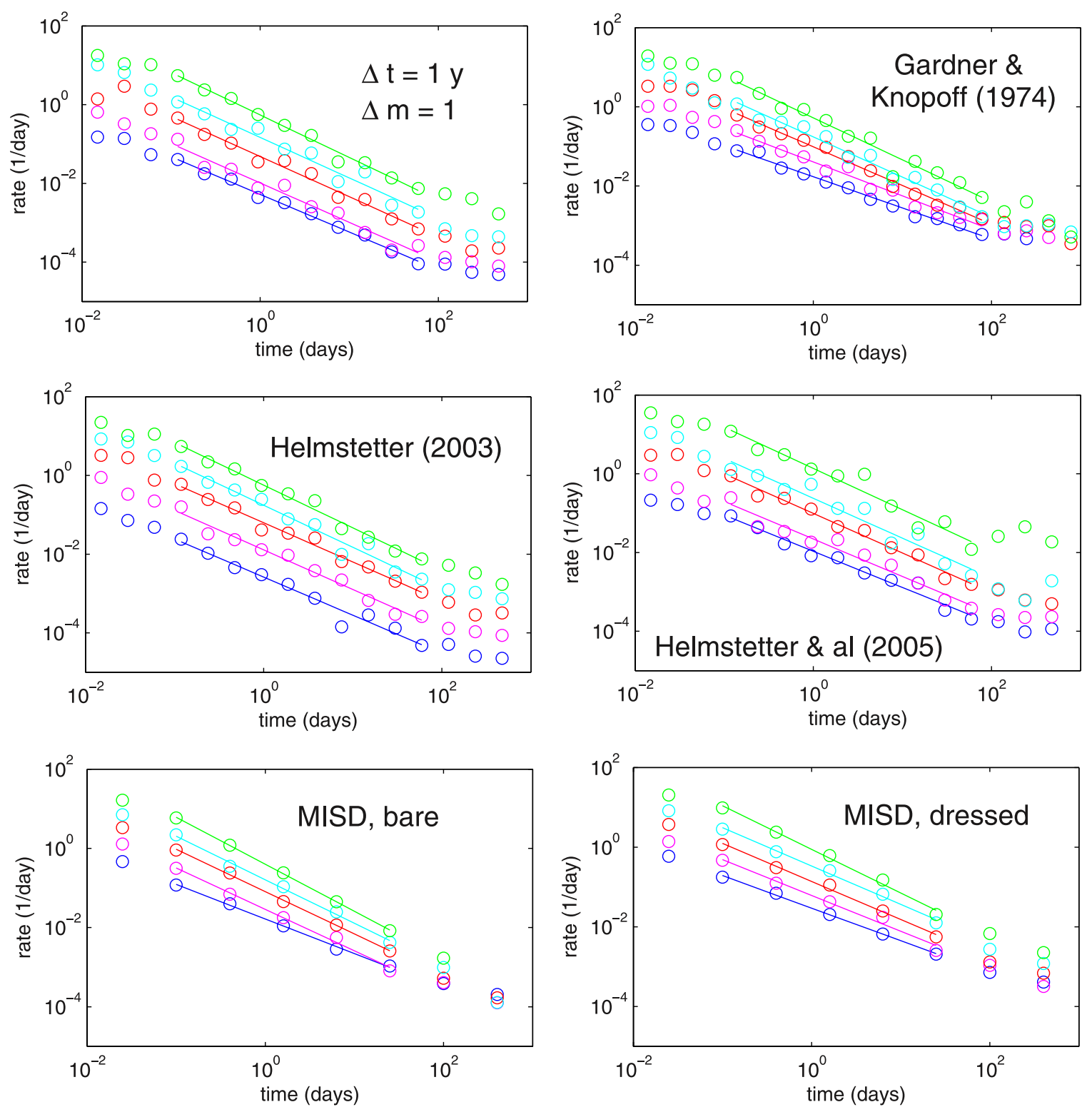

Figure 2. Aftershock rates following main shocks of magnitude $5.5 \leq m<6,6 \leq m<6.5,6.5 \leq m<7$, $7 \leq m<7.5$, and $m \geq 7.5$ (from bottom to top), using the various methods described in the text for selecting main shocks and aftershocks. Figure 2 (top left) corresponds to method 1 with $\Delta t=1$ year and $\Delta m=1$. The best power law fits performed in the interval between 0.1 day and 100 days are shown in continuous lines.

[9] 1. In a space-time window method, an earthquake $\{t, x, m\}$ is not a main shock if there exists too big and too close a previous earthquake $\left\{t^{\prime}, \underline{x}^{\prime}, m^{\prime}\right\}$, with $m^{\prime} \geq m-\Delta m$, at $t-\Delta t<t^{\prime}<t$ and their rupture zones overlap, i.e., $\left|\underline{x-x^{\prime}}\right|<$ $L(m)+L\left(m^{\prime}\right)$. The aftershocks of a main shock are all the earthquakes that follows it in its rupture zone, until a new main shock occurs which rupture zone overlaps with the current one. To test the sensitivity of the method to $\Delta m$ and $\Delta t$, we take either $\Delta t=1$ year, $\Delta m=1$, or $\Delta t=3$ years, $\Delta m=2$.

[10] 2. In the method by Helmstetter [2003], an earthquake $\{t, x, m\}$ is not a main shock if there exists a previous, larger earthquake $\left\{t^{\prime}, x^{\prime}, m^{\prime}>m\right\}$ within 1 year and $50 \mathrm{~km}$ independent of the magnitude. Then, all the earthquakes within a rupture length $L(m)$ and 1 year after the main shock are its aftershocks.

[11] 3. The method by Helmstetter et al. [2005] mimics the declustering algorithm of Reasenberg [1985]. Here, an earthquake $\{t, \underline{x}, m\}$ is not a main shock if there exists a previous earthquake $\left\{t^{\prime}, x^{\prime}, m^{\prime}\right\}$ with $m^{\prime} \geq m$-1 that occurred within 1 year and a distance $L\left(m^{\prime}\right)$. Then, an earthquake is an aftershock of a given main shock $\{t, x, m\}$ if it occurs within $L(m)$ and 1 year of it, or within $L\left(m^{\prime}\right)$ and 1 year of any of its previous aftershocks $\left\{t^{\prime}, \underline{x}^{\prime}, m^{\prime}\right\}$.

[12] 4. In the algorithm by Gardner and Knopoff [1974], an earthquake is an aftershock of a given main shock if it occurs within a time $T(m)$ and distance $R(m)$ of it, with both $T$ and $R$ increasing with $m$. We extend the magnitude range 
Table 1. Estimates for $p$ and $\chi$ Values $^{\mathrm{a}}$

\begin{tabular}{|c|c|c|c|}
\hline Magnitude & $p$ Value & $\chi$ Value & $r^{2}$ \\
\hline \multicolumn{4}{|c|}{ Method $1(\Delta t=1$ Year, $\Delta m=1)$} \\
\hline $5.5 \leq m<6$ & $0.96 \pm 0.01$ & $0.0052 \pm 0.0001$ & 0.99 \\
\hline $6 \leq \bar{m}<6.5$ & $1.00 \pm 0.05$ & $0.010 \pm 0.001$ & 0.96 \\
\hline $6 . \overline{5} \leq m<7$ & $1.02 \pm 0.01$ & $0.048 \pm 0.001$ & 0.98 \\
\hline $7 \leq m<7.5$ & $1.03 \pm 0.02$ & $0.15 \pm 0.01$ & 0.96 \\
\hline$m \geq 7.5$ & $1.08 \pm 0.01$ & $0.55 \pm 0.01$ & 0.99 \\
\hline \multicolumn{4}{|c|}{ Method $1(\Delta t=3$ Years, $\Delta m=2)$} \\
\hline $5.5 \leq m<6$ & $0.98 \pm 0.001$ & $0.0052 \pm 0.0001$ & 0.99 \\
\hline $6 \leq \bar{m}<6.5$ & $1.02 \pm 0.07$ & $0.010 \pm 0.001$ & 0.96 \\
\hline $6 . \overline{5} \leq m<7$ & $0.99 \pm 0.03$ & $0.042 \pm 0.002$ & 0.96 \\
\hline $7 \leq m<7.5$ & $1.13 \pm 0.03$ & $0.13 \pm 0.01$ & 0.94 \\
\hline$m \geq 7.5$ & $1.09 \pm 0.03$ & $0.40 \pm 0.02$ & 0.95 \\
\hline \multicolumn{4}{|c|}{ Method 2, Helmstetter [2003] } \\
\hline $5.5 \leq m<6$ & $0.97 \pm 0.01$ & $0.0026 \pm 0.0001$ & 0.97 \\
\hline $6 \leq \bar{m}<6.5$ & $1.00 \pm 0.04$ & $0.013 \pm 0.001$ & 0.98 \\
\hline $6 . \overline{5} \leq m<7$ & $0.99 \pm 0.01$ & $0.063 \pm 0.002$ & 0.99 \\
\hline $7 \leq m<7.5$ & $1.07 \pm 0.01$ & $0.18 \pm 0.01$ & 0.97 \\
\hline$m \geq 7.5$ & $1.08 \pm 0.01$ & $0.57 \pm 0.01$ & 0.99 \\
\hline \multicolumn{4}{|c|}{ Method 3, Helmstetter et al. [2005] } \\
\hline $5.5 \leq m<6$ & $0.92 \pm 0.01$ & $0.011 \pm 0.001$ & 0.98 \\
\hline $6 \leq m<6.5$ & $0.95 \pm 0.02$ & $0.022 \pm 0.001$ & 0.96 \\
\hline $6 . \overline{5} \leq m<7$ & $1.01 \pm 0.01$ & $0.10 \pm 0.01$ & 0.98 \\
\hline $7 \leq \bar{m}<7.5$ & $1.01 \pm 0.05$ & $0.24 \pm 0.02$ & 0.94 \\
\hline$m \geq 7.5$ & $1.05 \pm 0.03$ & $1.37 \pm 0.01$ & 0.95 \\
\hline \multicolumn{4}{|c|}{ Method 4, Gardner and Knopoff [1974] } \\
\hline $5.5 \leq m<6$ & $0.79 \pm 0.01$ & $0.017 \pm 0.001$ & 0.99 \\
\hline $6 \leq \bar{m}<6.5$ & $0.86 \pm 0.03$ & $0.041 \pm 0.001$ & 0.98 \\
\hline $6.5 \leq m<7$ & $0.98 \pm 0.01$ & $0.098 \pm 0.001$ & 0.99 \\
\hline $7 \leq \bar{m}<7.5$ & $1.02 \pm 0.02$ & $0.17 \pm 0.01$ & 0.98 \\
\hline$m \geq 7.5$ & $1.06 \pm 0.01$ & $0.53 \pm 0.02$ & 0.96 \\
\hline \multicolumn{4}{|c|}{ Method 5, Marsan and Lengliné [2008], Bare } \\
\hline $5.5 \leq m<6$ & $0.87 \pm 0.02$ & $0.017 \pm 0.001$ & 0.99 \\
\hline $6 \leq \bar{m}<6.5$ & $1.05 \pm 0.04$ & $0.029 \pm 0.001$ & 0.99 \\
\hline $6.5 \leq m<7$ & $1.07 \pm 0.01$ & $0.081 \pm 0.002$ & 0.99 \\
\hline $7 \leq \bar{m}<7.5$ & $1.10 \pm 0.02$ & $0.16 \pm 0.01$ & 0.99 \\
\hline$m \geq 7.5$ & $1.19 \pm 0.02$ & $0.40 \pm 0.01$ & 0.99 \\
\hline \multicolumn{4}{|c|}{ Method 5, Marsan and Lengliné [2008], Dressed } \\
\hline $5.5 \leq m<6$ & $0.82 \pm 0.02$ & $0.030 \pm 0.001$ & 0.99 \\
\hline $6 \leq \bar{m}<6.5$ & $0.90 \pm 0.07$ & $0.061 \pm 0.003$ & 0.98 \\
\hline $6.5 \leq m<7$ & $0.96 \pm 0.04$ & $0.14 \pm 0.01$ & 0.99 \\
\hline $7 \leq m<7.5$ & $0.96 \pm 0.05$ & $0.34 \pm 0.01$ & 0.99 \\
\hline$m \geq 7.5$ & $1.09 \pm 0.07$ & $0.88 \pm 0.04$ & 0.99 \\
\hline
\end{tabular}

${ }^{a}$ Values are of the best fits as shown in Figure 2, along with their errors, and the $r^{2}$ value giving the goodness of fit

of Gardner and Knopoff [1974] up to magnitude 9, by setting $T=1000$ days for $m \geq 8.5$ and keeping the $R \sim$ $10^{0.12 \mathrm{~m}}$ scaling. All the earthquakes that are not aftershocks are considered as main shocks.

[13] 5. The model-independent stochastic declustering (MISD) method by Marsan and Lengliné [2008] assumes that all the previous earthquakes have an influence on a subsequent earthquake, and that those influences sum up. The method then amounts to running an iterative algorithm converging toward the mean field influences (i.e., mean field in the sense that two earthquakes of equal magnitudes will be considered as having equal influences at the same interevent distances and times). This gives the bare (i.e., direct) influences. The dressed (i.e., both direct and indirect) influences are obtained by considering the full cascade of aftershocks triggering other aftershocks and so on, and summing over the various bare influences. There is no parameterization in this method. Notice that all the other methods exploited here only probe the dressed aftershock sequences.

[14] Figure 2 displays the aftershock rates for all these methods, along with the best power law fits $\lambda(t, m)=\chi \times t^{-p}$ which amounts to the Omori-Utsu law after neglecting the cutoff time $c$. These fits are computed for $0.1 \leq t \leq 100$ days (i.e., over 3 decades). No correction for the loss of aftershocks due to detection issues at short timescales is introduced. Given the quality of all the fits, we believe the scaling interval is appropriate for this "no-correction" choice, given these fitting time intervals. Table 1 summarizes the various estimates related to Figure 2.

[15] All the space-time window methods 1, 2, and 3 yield very similar rates. The method using the work by Gardner and Knopoff [1974] is also quite similar to the dressed rates of the MISD method. Although the general aspect is well preserved from one method to the other, with the notable exception of the bare rates using the MISD method (i.e., because all the other rates are dressed), subtle differences can however be seen. The parameters $p$ and $\chi$ obtained with the best fits are reported on Figure 3. As can be observed, there exists a significant dispersion of the parameters at all magnitudes, especially for the $p$ value, and even for one method by just changing its parameters (i.e., method 1, triangles). The $p$ values proposed by Ouillon and Sornette [2005] are occasionally significantly different from the ones obtained here. This could be due to the fact that they analyzed a very different data set than ours (southern California earthquakes), and also to the way they selected their time intervals for fitting the decays.

[16] The productivity is effectively found to follow a $\chi \sim$ $10^{\alpha m}$ scaling, although parameter $\alpha$ ranges between 0.66 (bare and dressed rates using MISD) and 1.15 (for most space-time window methods). This confirms that the productivity scaling is unfortunately strongly dependent on the selection method, as already discussed above. Low $\alpha$ values are typically obtained with ETAS inversions and the MISD method, which both perform space-time analyses and estimate the bare influences by assuming that the observed seismicity results from cascading. The other methods do not account for this cascading, and could therefore be biased toward large $\alpha$ values as a result. On the other hand, the inversion of cascading models with isotropic spatial kernel can lead to significant underestimation of the $\alpha$ parameter as recently shown for the space-time ETAS model [Hainzl et al., 2008].

[17] In the following, we will use the results shown in Figure 3 as a constraint for our model parameters. As there is yet no clear consensus on the "correct" values of $p$ and $\alpha$, we will ask our model to output values that are within the ranges shown in Figure 3 and proposed in past analyses, rather than attempting to reproduce one particular set of values.

\section{Model of Earthquake Occurrence}

[18] Many aftershocks occur on fault where quasi-static stress is expected to decrease after the main shock, resulting in an apparent paradox. However, earthquake slip is known to be heterogeneous, leading locally to an increased shear 

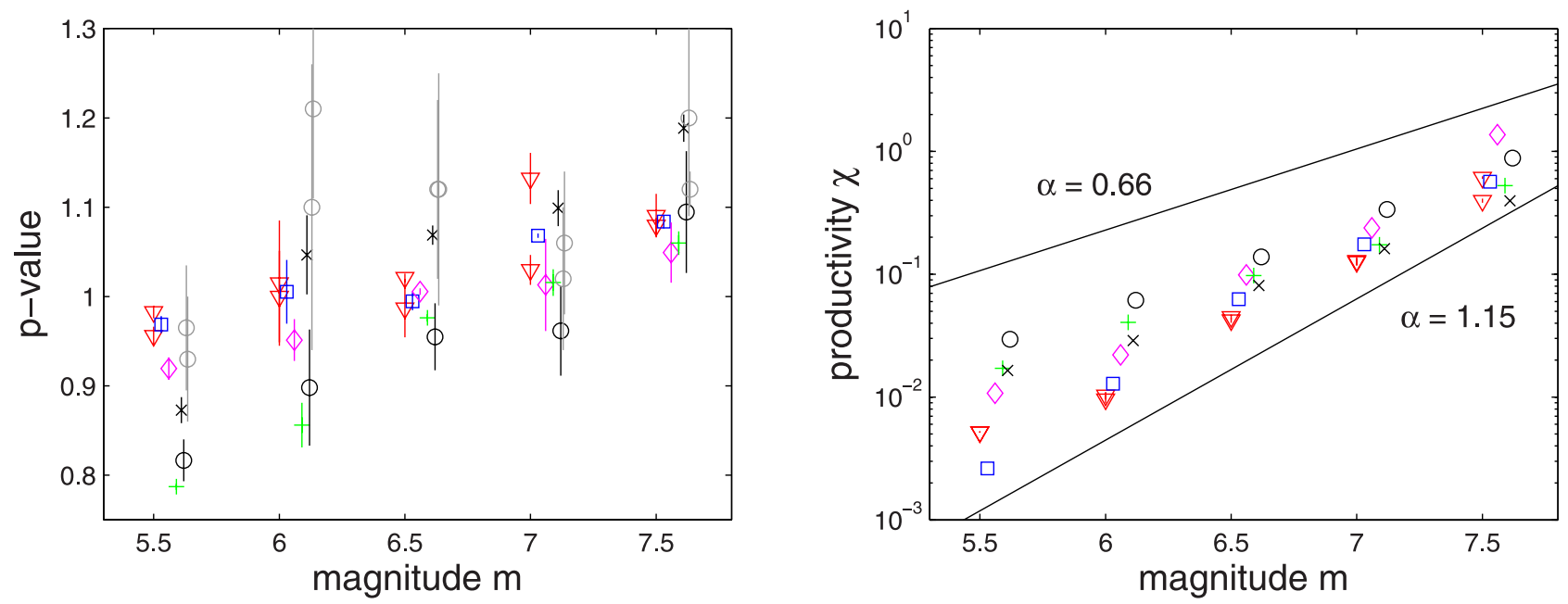

Figure 3. Parameters (left) $p$ and (right) $\chi$ of Omori-Utsu law obtained from the best fits shown in Figure 2. The symbols distinguish the various methods described in the text: method 1, triangles; method 2, squares; method 3, diamonds; method 4, pluses; method 5, dressed, circles; method 5, bare, crosses. For method 1, the two combinations using $\Delta t=1$ year, $\Delta m=1$ and $\Delta t=3$ years, $\Delta m=2$ are shown. The $p$ values obtained by Ouillon and Sornette [2005] for southern California earthquakes, using their two methods for selecting main shocks and aftershocks, are displayed for comparison (light gray). We added a small shift for presentation purposes. The two lines on Figure 3 (right) show a $10^{0.66 m}$ and a $10^{1.15 m}$ scaling. Table 1 summarizes all parameter estimates.

stress after main shock slip (i.e., loading rather than unloading). This has been observed by Mikumo and Miyatake [1995], Bouchon [1997], Bouchon et al. [1998], Day et al. [1998], Dalguer et al. [2002], Zhang et al. [2003], and Ripperger and Mai [2004] for a number of earthquakes. Examples of stress drop heterogeneity images at large scales are given by Day et al. [1998]. Heterogeneous fault stress has also been found in simulations by Parsons [2008] to be a long-lasting feature, with a spatial distribution of stress gaps showing persistence over tens of years.

[19] Recently, it has been shown that coseismic stress heterogeneities are able to explain aftershock activity, especially those observed in stress shadows such as within the main shock rupture [Helmstetter and Shaw, 2006; Marsan, 2006]. At the scale of the nucleation of seismic instability (typically meters to tens of meters as predicted by rate and state friction [cf. Dieterich, 1992, Figure 11]), the stress drop is dominated by this spatial variability: numerous nucleation patches are then loaded rather than unloaded by the main shock, resulting in the occurrence of aftershocks.

[20] As it is shown later, the situation is significantly different for smaller main shocks, i.e., characterized by rupture lengths not too large compared to the nucleation length. Then, scale invariance of the coseismic slip implies that the stress drop is much smoother (still at the scale of nucleation) than that of large main shocks. The ruptured fault is then mostly unloaded, and no aftershocks occur. The direct observation of this shadowing effect for small main shocks has been made by Rubin [2002], for relocated earthquakes on the San Andreas fault, and by Fischer and Horálek [2005] for relocated swarm earthquakes in the Vogtland area. In both cases, the stacked seismicity showed a significant gap within the rupture area.

[21] We here study how a model based on rate-and-state friction with a magnitude-dependent distribution of coseis- mic stress change can recover the aftershock decay characteristics described in section 2 .

\subsection{Description of the Model}

[22] In the following treatment, we postulate that (1) seismicity can be well described by the rate-and-state model of Dieterich [1994] with the ageing evolution law, in the localized nucleation regime for which healing is negligible [Rubin and Ampuero, 2005], (2) static stress triggering dominates the production of aftershocks, (3) the coseismic slip is fractal, causing the stress drop to be fractal as well, (4) spatial fluctuations in stress drop can be modeled with Gaussian statistics, (5) there exists a finite, time-independent nucleation length $\ell$ that characterize the size of fault patches self-accelerating to failure [Dieterich, 1992], and (5) all earthquakes initially nucleate at scale $\ell$, their final size being controlled by the dynamic propagation of the instability outside the nucleation patch rather than by processes occurring within this nucleation zone [Lapusta and Rice, 2003].

\subsubsection{Rate-and-State Model}

[23] According to Dieterich [1994], in the no-healing approximation, the seismicity rate $\lambda$ is inversely proportional to the state variable $\gamma$ describing the creep velocities on the faults, namely $\lambda(t)=\frac{r}{\dot{\tau} \gamma(t)}$, where $r$ is the stationary background rate of earthquakes and $\dot{\tau}$ the tectonic loading rate. The evolution of the state variable $\gamma$ is given by

$$
d \gamma=\frac{d t-\gamma d \tau}{A \sigma}
$$

with $A$ being a dimensionless fault constitutive parameter usually $\sim 0.01$ and $\sigma$ the effective normal stress. A sudden 

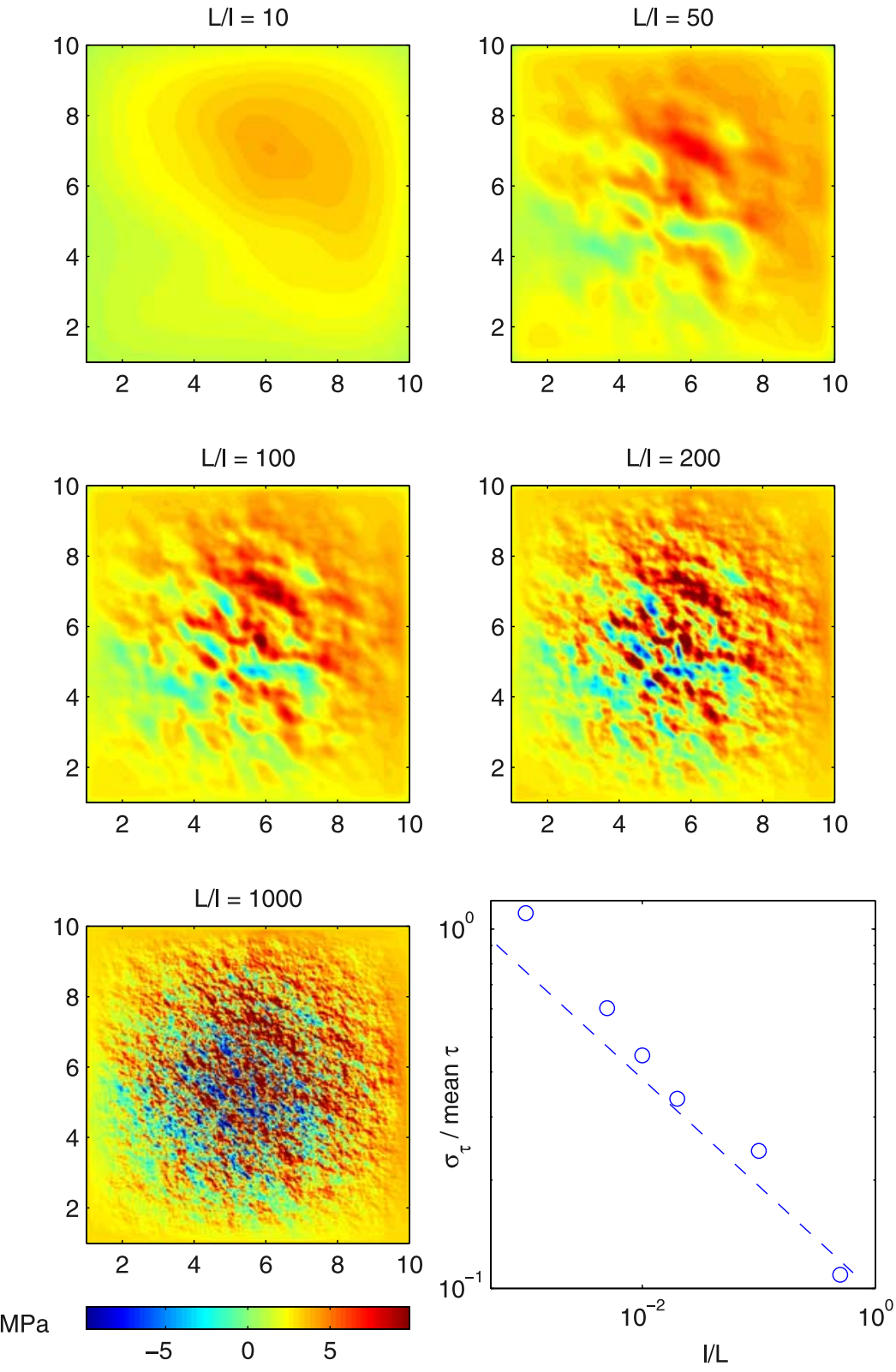

Figure 4. Stress drop distribution, in $\mathrm{MPa}$, on a $10 \times 10 \mathrm{~km}^{2}$ simulated fault, seen at a varying nucleation length $\ell$. The fractal stress field becomes rougher as the scale ratio $L / \ell$ grows. The mean stress drop is $3 \mathrm{MPa}$ whatever $l$. Stress loading corresponds to negative stress drops and is observed at places starting at $L / \ell=100$. (bottom right) Standard deviation $\sigma \tau$ normalized by the mean stress drop $3 \mathrm{MPa}$, function of the inverse ratio $\ell / L$. The power law trend (dashed line) follows the expected exponent $-1+$ $H=-0.3$ as predicted by equation (5). This can be compared to Figure 5 of Marsan [2006].

stress jump of $\tau$ for a background stationary rate $r$ leads to a time dependence of the activity according to

$$
\lambda(t, \tau)=\frac{r}{1+\left(e^{-\frac{\tau}{A \sigma}}-1\right) e^{-\frac{t}{t a}}}
$$

with $t_{a}=A \sigma / \dot{\tau}$. For simplicity, we will give hereinafter all stress jumps in units of $A \sigma$ and the time in units of $t_{a}$, unless stated otherwise, leading to the expression $\lambda(t, \tau)=r /[1+$ $\left.\left(e^{-\tau}-1\right) e^{-t}\right]$.

\subsubsection{Fractal Coseismic Slip and Stress Drop Heterogeneity}

[24] The stress variations induced by an earthquake are expected to be spatially heterogeneous due to coseismic slip as well as material heterogeneities. Thus, for any given crustal volume, the actual stress experienced by nucleation patches must be described by a probability density function $f(\tau)$, and the earthquake activity of the volume must be calculated by

$$
\lambda(t)=\int \lambda(t, \tau) f(\tau) d \tau
$$




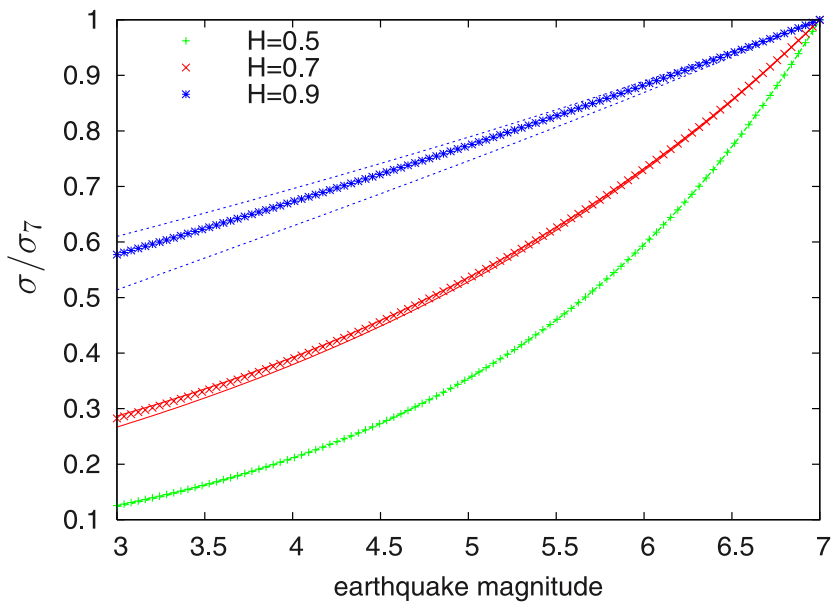

Figure 5. The dependence of the expected stress drop variability $\sigma / \sigma_{7}$ on the main shock magnitude $m$ for Hurst exponents inverted from observations. For each Hurst exponent, the symbols refer to $m_{0}=-2$, while the lines refer to $m_{0}=-4$ and $m_{0}=0$, respectively. We use $\beta=0.45$ in the $L \sim 10^{\beta \times m}$ relation, according to Wells and Coppersmith [1994].

On or close to the main fault, stress heterogeneity is dominated by slip variability. Scale-invariant slip models have been proposed by several authors [Andrews, 1980; Frankel, 1991; Herrero and Bernard, 1994; Mai and Beroza, 2002]. For a two-dimensional fractal model, the slip $u(k)$ is proportional to $k^{-1-H} g(k)$ with $H$ the Hurst exponent related to the fractal dimension $D=3-H$, where $g$ is a realization of a Gaussian white noise, and $k$ the wave number. In their extended analysis of the slip distributions of 44 earthquakes, Mai and Beroza [2002] found that $H=$ $0.71 \pm 0.23$. Since the stress drop scales as $\tau(k) \sim k u(k) \sim$ $k^{-H} g(k)$ [e.g., see Schmittbuhl et al., 2006], the scaling of the standard deviation $\sigma_{\tau}$ of the stress change at the length scale of the nucleation sites, $\ell$, is given by Marsan [2006]:

$$
\sigma_{\tau}=C \sqrt{\left(\frac{L}{\ell}\right)^{2-2 H}-1}
$$

for $H<1$, where $L$ is the rupture length of the earthquake. The standard deviation, hence the variability of the stress drop, thus diverges for $\ell \rightarrow 0$ when $H \leq 1$ [Helmstetter and Shaw, 2006]. Figure 4 shows an example of how the stress drop roughness depends on the scale ratio between the fault size $L$ and the nucleation scale $\ell$. A $10 \times 10 \mathrm{~km}^{2}$ fault is simulated, which roughly corresponds to a magnitude 6 earthquake: we generate a fractal (scalar) slip $u(x, y)$ with Hurst exponent $H=0.7$, such that the stress drop, defined as $\left(\partial_{x}+\partial_{y}\right) u$, has a mean value of $3 \mathrm{MPa}$. We vary the scale of observation, thus changing the scale ratio between the rupture size $L$ and the cutoff scale $\ell$. As this scale ratio is increased, the roughness of the stress drop is enhanced, with the emergence of patches undergoing stress loading (i.e., negative stress drops).

[25] We calibrate the intensity of the stress fluctuation by considering that the induced stress variability of large earthquakes is typically of the order of the average stress drop $\bar{\tau}$, when observed at the $\simeq 5 \mathrm{~km}$ scale. Using $H=0.7$, this gives that the stress variability at the nucleation length scale of approximately $10 \mathrm{~m}$ would be of the order of $6 \bar{\tau}$. In the following, we define the stress variability $\sigma_{7}$ induced by a $m=7$ event as an input parameter, typically ranging between 0.1 and 10 times the mean stress drop: $0.1<\mathrm{CV}=$ $\sigma_{7} / \bar{\tau}<10$, where $\mathrm{CV}$ is the coefficient of variation of the stress distribution.

[26] The dependence of the stress drop heterogeneity on the magnitude is given by equation (5), together with $L=\ell 10^{\beta(m-m)}$, where the empirical value of $\beta$ is close to 0.45 [Wells and Coppersmith, 1994] (assuming the rupture length as the square root of the rupture area). We denote by $m_{0}$ the magnitude corresponding to a rupture size of $\ell$, i.e., the minimum magnitude for frictioncontrolled earthquakes.

\subsubsection{Stress Drop Modeled With Gaussian Statistics}

[27] A Gaussian model for $\tau$ is only a first-order approximation. There is evidence for an asymmetric stress drop in some instances [Day et al., 1998], with pronounced peaks of high stress drop embedded in large zones of low, negative stress drop. Elaborating even further away from a Gaussian model, Lavallée and Archuleta [2003, 2005] have proposed that the slip distribution of both the 1979 Imperial Valley and the 1999 Chi-Chi earthquakes are better modeled by Lévy stable statistics. In this model, $\tau(k) \sim k^{-H} g_{\alpha}(k)$, where $g_{\alpha}$ is a Lévy noise with stability index $\alpha$, typically with $\alpha$ close to 1 (hence $g_{\alpha}$ close to a Cauchy noise). The difficulty in handling this type of model is that the stress drop distribution can no longer be characterized by its standard deviation, as it is not defined anymore. Clearly, Lévydistributed stress drops will generate even rougher fields, and the results presented in this paper, that are based on normal (Gaussian) laws, can therefore be seen as a "mostconservative," i.e., least heterogeneous, limit case.

\subsubsection{Nucleation Size}

[28] So far, nucleation zones lack direct observation. Therefore, we assume the simplest case that the nucleation size is independent of the aftershock magnitude. This is in agreement with the well-known cascade model for earthquake ruptures [e.g., Kilb and Gomberg, 1999] and numerical simulations [Lapusta and Rice, 2003]. In particular, we assume that the magnitude $m_{0}$, which corresponds to the nucleation size $\ell$, is constant. In the case of $m_{0}(m)$, our results would be directly applicable only for each magnitude band of the aftershocks separately. However, because of the weak dependence of our results on $m_{0}$ (see Figure 5), our general results are expected to remain valid even in this case.

\subsection{Model Predictions Versus Observations}

[29] We calculate the seismicity rate within the rupture zone of the main shock by solving equation (4) numerically with a magnitude-dependent Gaussian probability distribution, i.e., $f(\tau)$ is Gaussian with mean $-\bar{\tau}$ and standard deviation $\sigma_{\tau} . \bar{\tau}$ is the (Coulomb) stress drop on the main fault which can be seen further away from the fault [King and Cocco, 2001; Freed, 2005; Steacy et al., 2005]: adding stress heterogeneities allows to go beyond usual Coulomb stress modeling by introducing a stochastic term to the deterministic stress field. This stochastic term is here viewed as accounting for the small-scale variability that is 
Table 2. Summary of the Model Parameters That Affect the Aftershock Decay Characteristics

\begin{tabular}{|c|c|c|}
\hline Parameter & Description & Value \\
\hline$A \sigma$ & $\begin{array}{l}\text { constitutive parameter } \\
\text { times effective normal stress }\end{array}$ & $0.1 \mathrm{MPa}$ \\
\hline $\bar{\tau}$ & $\begin{array}{l}\text { mean stress drop on } \\
\text { the main fault (the negative } \\
\text { of the mean stress change) }\end{array}$ & variable \\
\hline$\sigma_{\tau}$ & $\begin{array}{l}\text { stress drop standard deviation } \\
\text { at the nucleation length scale }\end{array}$ & variable \\
\hline$\ell, m_{0}$ & $\begin{array}{l}\text { nucleation length }(\ell) \text { and } \\
\text { equivalent nucleation } \\
\text { magnitude }\left(m_{0}\right)\end{array}$ & $\ell=2.2 \mathrm{~m}, m_{0}=-2$ \\
\hline$C, \sigma_{7}, \mathrm{CV}$ & $\begin{array}{l}\text { calibration constants for } \sigma_{\tau} \text {, } \\
\text { see equation }(5) ; \sigma_{7}=\sigma_{\tau} \text { for } \\
m=7 \text { earthquakes, } \\
\text { and } \mathrm{CV}=\sigma_{7} / \tau\end{array}$ & variable \\
\hline$H$ & $\begin{array}{l}\text { Hurst exponent of fractal } \\
\text { slip distribution }\end{array}$ & $0.7 \pm 0.2$ \\
\hline
\end{tabular}

not accessible to direct measurement nor computation. It can alternatively be seen as modeling the error on the largescale stress field: as well as a mean stress drop $\bar{\tau}$, we also need its uncertainty $\sigma_{\tau}$. Accounting for such an uncertainty is not a second-order refinement: as already shown by Helmstetter and Shaw [2006] and Marsan [2006], it can significantly alter the seismicity.

[30] The model has a number of parameters, which have a direct influence on the Omori-Utsu parameters $p$ and $\chi$. We summarize these parameters in Table 2, along with their values. For an earthquake of magnitude $m$, the distribution of stress drops on the main fault is thus a Gaussian distribution with mean $\bar{\tau}$ independent of $m$, and standard deviation as given by equation (5). The crucial point here is that this standard deviation increases with the magnitude $m$, this increase being constrained by parameters $C$ (or equivalently $\sigma_{7}$ or $\mathrm{CV}$ ), $\ell$ (or $m_{0}$ ), and $H$. Changing these three

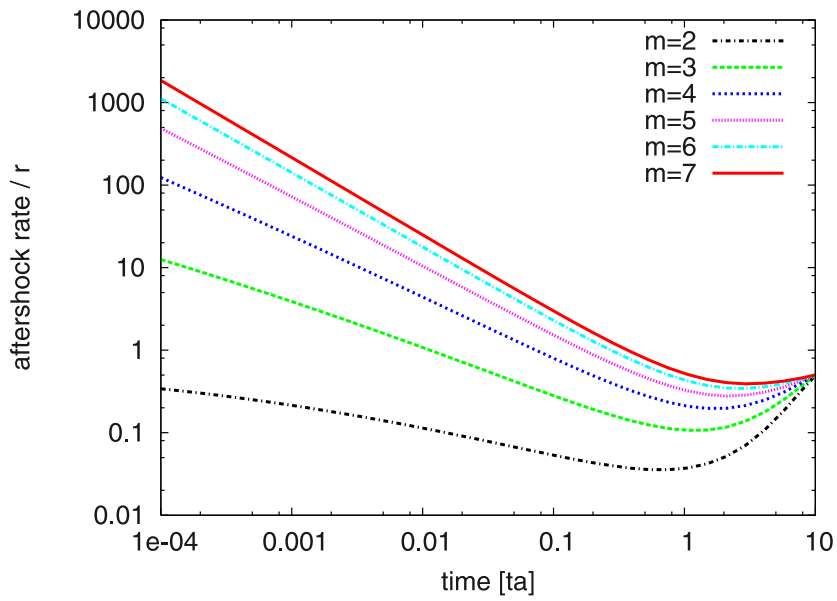

Figure 6. The aftershock decay as a function of the main shock magnitude in the case of $H=0.7, \mathrm{CV}=\sigma_{7} / \bar{\tau}=2.3$, and $\bar{\tau}=1 \mathrm{MPa}$. The aftershock rate is normalized by the background rate. At long timescales (i.e., $t / t_{a}$ typically greater than 1), the aftershock rate becomes less than the background rate, indicating the onset of a seismic quiescence. This is caused by the overall stress drop [see Marsan, 2006]. key parameters amounts to changing the dependence of $p$ and $\chi$ on $m$.

[31] The standard value of the Hurst exponent $H$ is set to 0.7 because it was the mean value obtained by Mai and Beroza [2002]. Letting $H$ vary within the acceptable range $0.5 \leq H \leq 0.9$ strongly affects the results, as decreasing $H$ causes the stress field to become more heterogeneous. This is further discussed in section 3.4.

[32] A first point is to note that the aftershock decay depends very little on $\ell$ and $m_{0}$, as long as they remain very small compared to the sampled rupture lengths and magnitudes. Figure 5 illustrates the increasing stress drop heterogeneity for increasing earthquake magnitudes for the range of Hurst exponents inverted from slip data and for three different $m_{0}$ value. The tested values of $m_{0}=-4,-2$, and 0 correspond to nucleation length of approximately $0.3 \mathrm{~m}$, $2.2 \mathrm{~m}$, and $18 \mathrm{~m}$ [Wells and Coppersmith, 1994]. Given that the dependence on the assumed $m_{0}$ value is very weak, we (arbitrarily) set $m_{0}$ to -2 for the remainder of this study.

[33] The only parameters left to vary are therefore the mean stress drop and the calibration constant $C$ (or $\sigma_{7}, \mathrm{CV}$ ). We calculate the aftershock rate as a function of the main shock magnitude for different values of these parameters. Figure 6 shows the aftershock decay for different main shock magnitudes in the case of a coefficient of variation $\mathrm{CV} \equiv \sigma_{7} / \bar{\tau}=2.3$ and a stress drop of $\bar{\tau}=1 \mathrm{MPa}$.

[34] The Omori-Utsu law is fitted to each of these curves in the time interval $\left[10^{-4}-10^{-1}\right]$, yielding an estimate of the $p$ value as a function of the main shock magnitude. For a stress field variation of $\mathrm{CV}=2.3$, the magnitude dependence is found to be in good agreement with the observed $p$ value dependence in California [Ouillon and Sornette, 2005] and to our global analysis of section 2. This is shown in Figure 7. Note that for significantly stronger heterogeneities, the magnitude dependence becomes quite weak and would be difficult to detect in real data (see the curve for $\mathrm{CV}=8.0$ in Figure 7): in this case, the stress heterogeneity

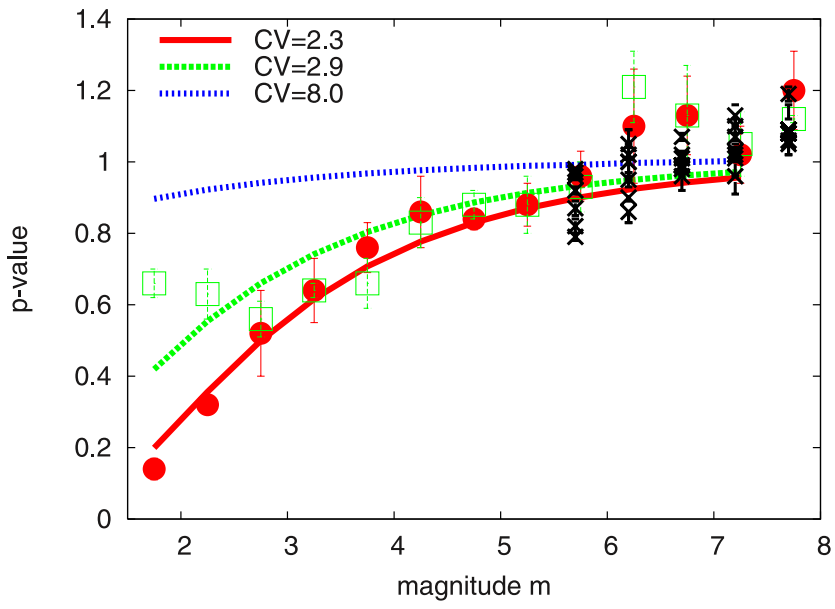

Figure 7. The $p$ value as a function of the main shock magnitude in the case of $H=0.7$ and $\mathrm{CV}=\sigma_{7} / \bar{\tau}=2.3,2.9$, and 8.0. The curves are compared with the observed $p$ value dependence in California (data from Ouillon and Sornette [2005], results based on their declustering method 1 (dots) and declustering method 2 (squares)) and with the range of $p$ values reported in section 2 (crosses). 


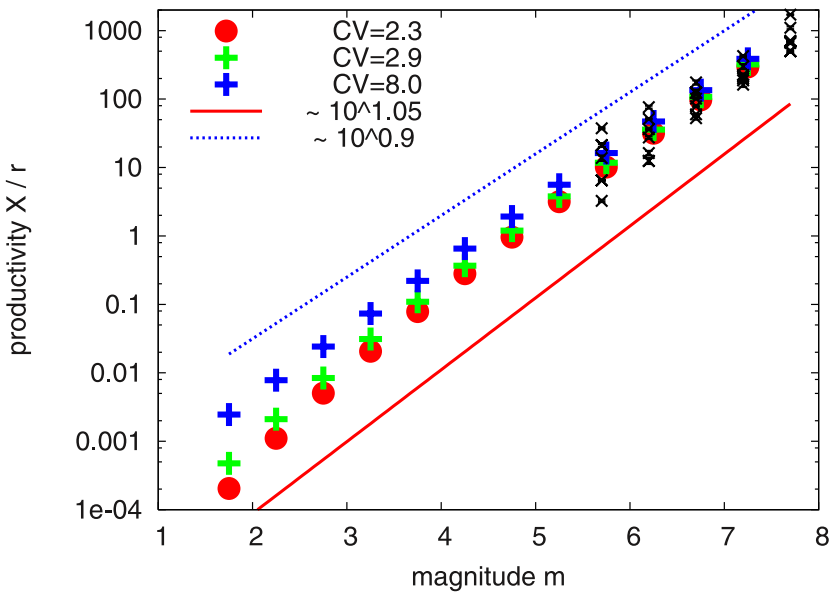

Figure 8. The aftershock productivity as a function of main shock magnitude. Note that the productivity is normalized by the background rate $r$. The results are compared with the two scaling laws $\sim 10^{1.05 m}$ and $\sim 10^{0.9 m}$. For a comparison, the observational $\chi$ values reported in section 2 have to be rescaled by the unknown factor $t_{a} / r\left(t_{a}\right.$ measured in units of days). For a factor of 1250, the observations are represented by small crosses.

is large enough even at magnitude 2 to push the $p$ value close to 1 .

\subsection{Aftershock Productivity as a Function of Main Shock Magnitude}

[35] In the case that ruptures produce a stress drop variability which is independent of the earthquake magnitude, our model would predict an aftershock productivity which would simply scale with the main shock rupture area, i.e., $\sim 10^{0.9 m}$. However, as another consequence of the scaling of stress heterogeneity with main shock magnitude, the aftershock productivity is not simply scaling with main shock area anymore. For the previous examples, the productivity values $\chi$ are shown in Figure 8 . For moderate stress heterogeneities, the increase of the aftershock productivity is close to $\sim 10^{1.05 m}$ which is the empirical scaling exponent found by Helmstetter et al. [2005] for California and in agreement with our own investigations of the global earthquake catalog with methods 1-3 (Figure 3).

[36] For significantly larger heterogeneities $(\mathrm{CV}=8.0)$, the scaling exponent is smaller and becomes almost $\sim 10^{0.9 \mathrm{~m}}$. Thus for large heterogeneities, the model predicts an almost constant $p \approx 1$ value and an aftershock productivity which simply scales with the rupture area.

[37] We have implicitly assumed that aftershocks can occur everywhere on and close to the main shock fracture. However, some studies indicate that aftershocks occur on spatial fractals with dimension $D<2$ [Turcotte, 1997; Helmstetter et al., 2005]. Assuming that aftershocks are restricted to such fractal subsets of the fault plane, we would get a smaller theoretical cutoff value $\alpha_{\min }=\beta \cdot D=0.45 \mathrm{D}$ instead of 0.9 .

\subsection{Dependence on the Hurst Exponent and the Stress Drop}

[38] Our general findings are independent of the assumed value of the mean stress drop $\bar{\tau}$. The increase of the $p$ value and the aftershock productivity is found to be preserved for other values of $\bar{\tau}$. However, changing $\bar{\tau}$ impacts on the degree of the stress field heterogeneity which is needed to produce the same magnitude dependence. For example, practically the same curve as shown in Figure 7 for $\bar{\tau}=$ $1 \mathrm{MPa}$ and $\mathrm{CV}=2.3$ is found for $\bar{\tau}=0.5 \mathrm{MPa}$ with $\mathrm{CV}=$ 4.0 and $\bar{\tau}=2 \mathrm{MPa}$ with $\mathrm{CV}=1.6$. These results depend also on the Hurst exponent. Figure 9 shows for the case of $\bar{\tau}=$ $1 \mathrm{MPa}$ the same characteristics for the lower and upper limits of the observed Hurst exponents, $H=0.5$ and $H=0.9$. In each case, the standard deviation $\sigma_{7}$ is chosen such that the $p$ value dependence on magnitude fits the observation best. It is found that higher Hurst exponents underestimate the observed magnitude dependence whereas lower Hurst exponents seem to overestimate the trend. Thus the value $H=0.7$ which is independently found to best describe (a)

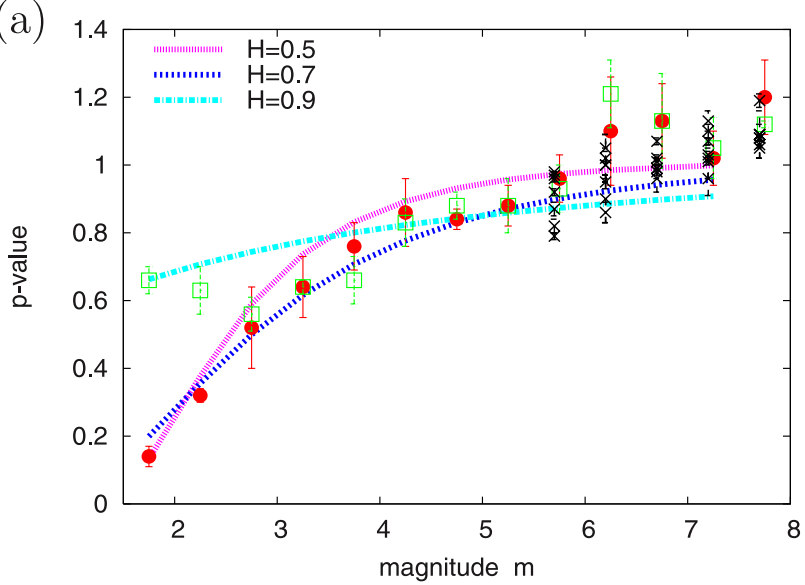

(b)

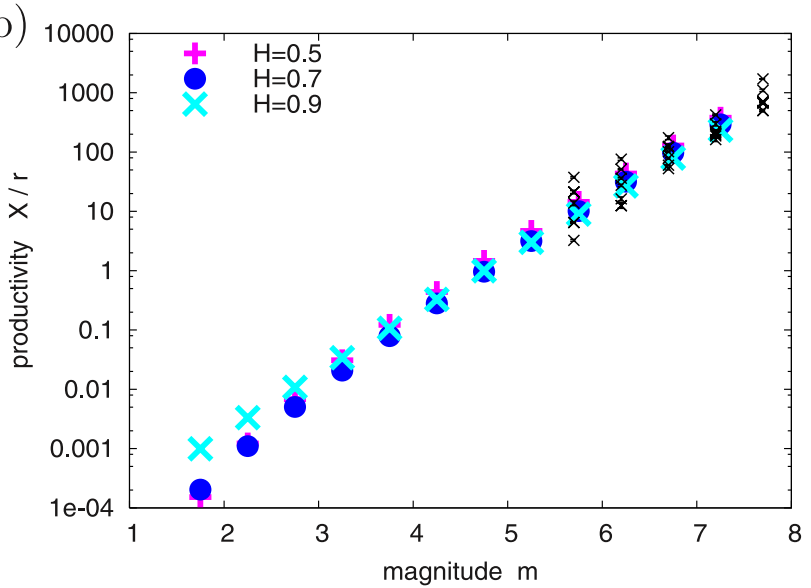

Figure 9. (a) The $p$ value as a function of the main shock magnitude in comparison for the three different values $H=0.5,0.7$, and 0.9 . In each case the stress field heterogeneity is chosen in a way that the empirically data points (for description, see Figure 7) are best fitted: $\mathrm{CV}=6.5$ for $H=0.5$; $\mathrm{CV}=2.3$ for $H=0.7$, and $\mathrm{CV}=1.5$ for $H=0.9$. (b) The aftershock number as a function of main shock magnitude for the same cases. In both cases, the data reported in section 2 are added (small crosses). 


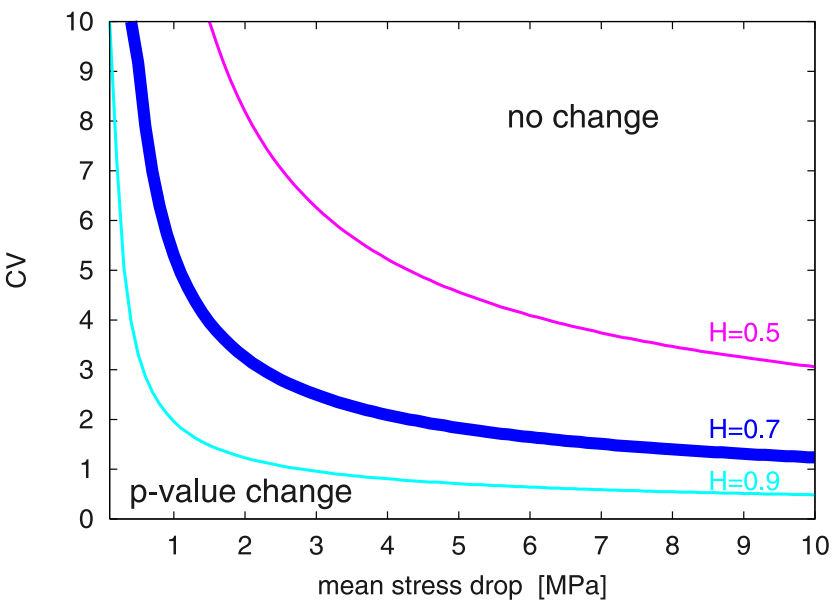

Figure 10. Phase diagram for significant $p$ value changes. The plot shows the stress field variability $\mathrm{CV}$ which leads to a $p$ value increase of 0.1 from $m=3.0$ to 7.0 main shocks, considered as an observable change: For lower $\mathrm{CV}$ values, the $p$ value change with main shock magnitude is significant, while $p$ value changes could be hardly detected in empirical data sets for higher CV values.

observed slip distributions is also found to give consistently the best description of the aftershock decay. This is another indication of the applicability of the rate-and-state friction model for aftershocks.

[39] To examine the whole parameter space more systematically, we calculate, for stress drops varying between $0.1 \mathrm{MPa}$ and $10 \mathrm{MPa}$, the stress field variability $\mathrm{CV}$ which leads to a $p$ value increase of 0.1 from $m=3.0$ to 7.0 main shocks. The resulting curves are shown for the different Hurst exponents in Figure 10. These curves can be seen as the boundary delineating the parameter region where significant $p$ changes should be detectable from the analysis of empirical data sets: For lower CV values, the $p$ value change is larger than 0.1 while, for higher $\mathrm{CV}$ values, $p$ value changes (smaller than 0.1 ) could be hardly detected in empirical data sets. It is found that for the same CV value, the $p$ value change becomes more significant for smaller stress drop values.

\section{Influence of Afterslip on the $p$ and $\chi$ Dependence on $m$}

[40] There is growing evidence that large main shocks are followed by significant amounts of afterslip [e.g., Miyazaki et al., 2004; Chlieh et al., 2007]. It has been proposed that this afterslip, which typically decays as $1 / t$ (see Montési [2004] for analysis and modeling of afterslip decay), could be the driving force in producing aftershocks [Perfettini and Avouac, 2004]. Dieterich [1994] derived, in the context of rate-and-state friction, the earthquake rates that would be triggered by a $1 / t$-decaying afterslip following a coseismic stress change. Addition of afterslip is indeed seen to substantially modify the aftershock decay, both in terms of decay exponent ( $p$ value) and of aftershock productivity. We therefore consider in this section how afterslip could further change the conclusions reached in section 3 .
[41] For a coseismic stress change $\tau$ followed by an afterslip-induced stress of the form

$$
\tau_{1} \times \ln \left(1+t / t^{*}\right),
$$

solving equation (2) leads to the seismicity rate

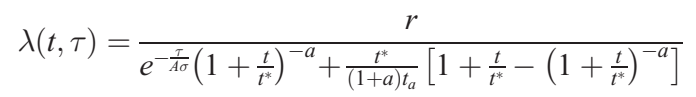

in place of equation (3) [see Dieterich, 1994]. Parameter $a$ equals $\tau_{1} / A \sigma$. This solution ignores the constant, tectonic stressing rate $\dot{\tau}$ contribution to the postseismic stress. Accounting for it affects the aftershock decay $\lambda(t, \tau)$ (as given by equation (7)) only when $t$ becomes comparable to $t_{a}$, and amounts to a convergence of the rate to the background rate $r$. As an illustration, Figure 11 compares the solution of equation (7) that ignores the tectonic loading, with the numerical solution of equation (2) that includes this loading.

[42] We analyze the effect of afterslip on the $p$ value variations and the aftershock productivity for the previous example of $\bar{\tau}=1 \mathrm{MPa}$ and $\mathrm{CV}=2.3$. Parameter $t^{*}$ is set to $10^{-7} t_{a}$. The strength of the stress changes induced by afterslip is characterized by the ratio between the cumulative stress change by afterslip within time $t_{a}$ and the mean of the coseismic stress drop $\bar{\tau}$. The results are shown in Figure 12. For additional loading (positive values of $\tau_{1}$ ), the $p$ values slightly decrease and the productivity increases. Vice versa for an unloading (negative values of $\tau_{1}$ ): $p$ values increase and the productivity decreases. $p$ values larger than 1 are found in the case of very strong unloading when the afterslip induced stress is of the order of the coseismic mean stress drop. However, in all cases, the consideration of afterslip does not change the general shape of both the

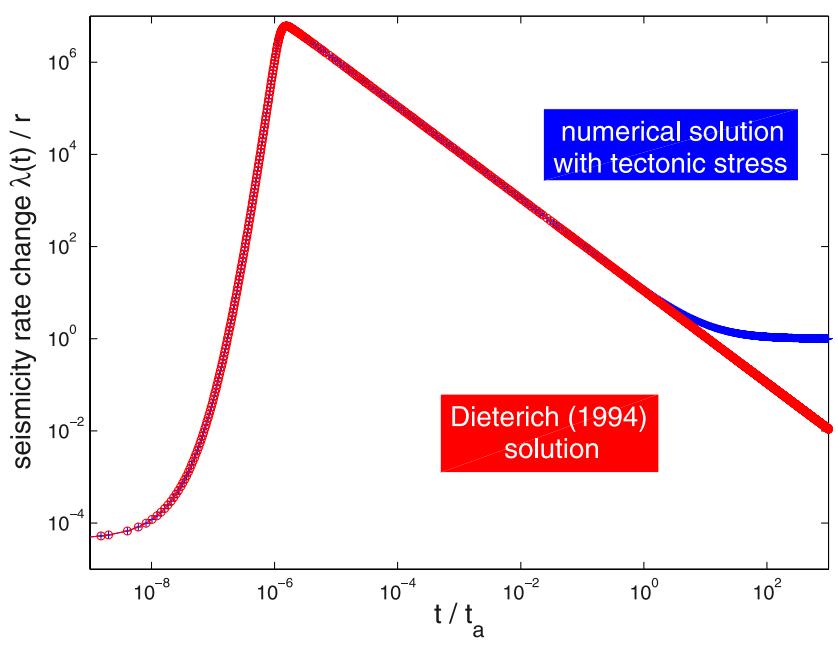

Figure 11. Seismicity rate normalized by background rate, for a $1 \mathrm{MPa}$ coseismic stress drop followed by a stress increase due to afterslip $\tau_{1} \times \ln \left(1+t / t^{*}\right)$. Here $\tau_{1}=1 \mathrm{MPa}$, $t^{*} / t_{a}=10^{-7}, A \sigma=0.1 \mathrm{MPa}$, and the tectonic stress rate is $\dot{\tau}=0.1 \mathrm{MPa}$ per unit $t_{a}$. Both solutions are identical as long as $t<t_{a}$. 

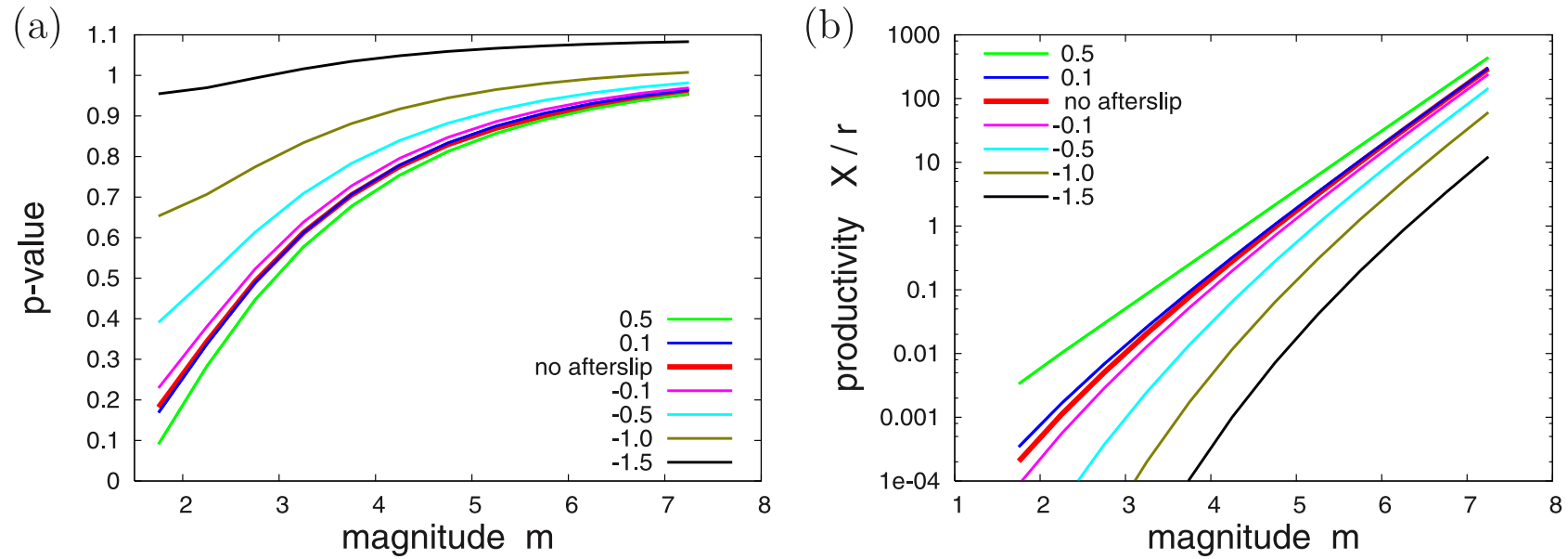

Figure 12. Consideration of postseismic stress changes due to afterslip: (a) the $p$ value and (b) the productivity as a function of the main shock magnitude. The number for each curve gives the fraction of the coseismic mean stress drop which is added (positive sign for loading and negative sign for unloading) within time $t_{a}$ to the coseismic stress value according to equation (6). All curves are based on $\bar{\tau}=1 \mathrm{MPa}$ and $\mathrm{CV}=2.3$.

$p$ value change and the scaling of the productivity with main shock magnitude.

\section{Summary and Conclusions}

[43] In this paper, we investigate the main shock magnitude dependence of the aftershock activity which results from fractal slip and frictional nucleation of earthquakes. Fractal earthquake slip directly leads to a rupture sizedependent heterogeneity of the induced stress field. The larger the earthquake, the stronger is the expected variance of the stress changes. Thus larger earthquakes will typically produce strongly loaded patches within the rupture zone, even though the average stress level dropped significantly. In such loaded patches, which are for smaller events less frequent, aftershocks will nucleate rapidly. We systematically studied the predicted aftershock characteristics and compared them with observations. First, the model predicts that small earthquakes should be followed by an immediate on-fault seismicity shadow, while larger earthquakes should not because of the induced stress drop heterogeneity. Direct observation of this shadowing effect for small main shocks has been made by Rubin [2002], for relocated earthquakes on the San Andreas fault, and by Fischer and Horlek [2005] for relocated swarm earthquakes in the Vogtland area. Second, Omori-Utsu's $p$ value increases with main shock magnitude as a consequence of enlarged stress field heterogeneity. The aftershock productivity $\chi$ is also affected, although less significantly, by the stress heterogeneity: its scaling $\chi \sim 10^{\alpha m}$ with main shock magnitude $m$ is made steeper by a rough stress field ( $\alpha \simeq 1.05$ compared to $\alpha=0.9$ when there is no heterogeneity). Both predictions are in good agreement with recent observations by Ouillon and Sornette [2005] and Helmstetter et al. [2005] and our own observations of section 2. In particular, we find that the Hurst exponent deduced from slip inversions, $H=0.7$, gives the best fit to the data which supports the model.

[44] To prove the robustness of the recently observed main shock magnitude dependence of the $p$ value and the scaling of the aftershock productivity $\chi$, we have performed an independent analysis of the global earthquake catalog for main shock magnitudes $M \geq 5.5$. For a number of different declustering algorithms, we could confirm a systematic increase of the $p$ value with main shock magnitude. On the other hand, we found that the apparent productivity value is strongly dependent on the selection algorithm, resulting in a broad interval of possible values between 0.6 and 1.15. By means of a systematic parameter analysis, we have used these empirical observations to constrain the expected degree of the stress drop variability.

[45] Within the same model framework, $p$ values larger than 1 cannot be explained if only coseismic main shockinduced stress changes and tectonic loading are considered. This is in contradiction with empirical observations of $p>1$ aftershock decays. However, Dieterich [1994] already showed that $\log (t)$ unloading in agreement with frequently observed afterslip can explain $p>1$. We have checked numerically that this result remains true if tectonic forcing is additionally taken into account. Our analysis shows, however, that afterslip does not change the general characteristics of the main shock-magnitude dependence of the $p$ value and the aftershock productivity.

[46] Acknowledgments. We are grateful to Rodolfo Console, Sandy Steacy, and an anonymous referee for helpful recommendations. S.H. was supported by the EU-SAFER project (contract 036935).

\section{References}

Andrews, D. J. (1980), A stochastic fault model: 1. Static case, J. Geophys. Res., 85, 3867-3877.

Bouchon, M. (1997), The state of stress on some faults of the San Andreas system as inferred from near-field strong motion data, J. Geophys. Res., 102, 11,731-11,744.

Bouchon, M., M. Campillo, and F. Cotton (1998), Stress field associated with the rupture of the 1992 Landers, California, earthquake and its implication concerning the fault strength at the onset of the earthquake, J. Geophys. Res., 103, 21,091-21,097.

Chlieh, M., et al. (2007), Coseismic slip and afterslip of the great $M_{w} 9.15$ Sumatra-Andaman earthquake of 2004, Bull. Seismol. Soc. Am., 97, $152-173$. 
Console, R., M. Murru, and A. M. Lombardi (2003), Refining earthquake clustering models, J. Geophys. Res., 108(B10), 2468, doi:10.1029/ 2002JB002130.

Dalguer, L. A., K. Irikura, W. Zhang, and J. D. Riera (2002), Distribution of dynamic and static stress changes during 2000 Tottori (Japan) earthquake: Brief interpretation of the earthquake sequences; foreshocks, mainshock and aftershocks, Geophys. Res. Lett., 29(16), 1758, doi:10.1029/ 2001GL014333.

Day, S. M., G. Yu, and D. J. Wald (1998), Dynamic stress changes during earthquake ruptures, Bull. Seismol. Soc. Am., 88, 512-522.

Dieterich, J. H. (1992), Earthquake nucleation on faults with rate- and statedependent friction, Tectonophysics, 211, 115-134.

Dieterich, J. H. (1994), A constitutive law for rate of earthquake production and its application to earthquake clustering, J. Geophys. Res., 99, 26012618.

Enescu, B., J. Mori, and M. Miyazawa (2007), Quantifying early aftershock activity of the 2004 mid-Niigata Prefecture earthquake $\left(M_{w} 6.6\right), J$. Geophys. Res., 112, B04310, doi:10.1029/2006JB004629.

Felzer, K. R., R. E. Abercrombie, and G. Ekstrom (2004), A common origin for aftershocks, foreshocks, and multiplets, Bull. Seismol. Soc. Am., 94, $88-98$.

Fischer, T., and J. Horálek (2005), Slip-generated patterns of swarm microearthquakes from West Bohemia/Vogtland (central Europe): Evidence of their triggering mechanism?, J. Geophys. Res., 110, B05S21, doi:10.1029/ 2004JB003363.

Frankel, A. (1991), High-frequency spectral falloff of earthquakes, fractal dimension of complex rupture, $\mathrm{b}$ value, and the scaling of strength on fault, J. Geophys. Res., 96, 6291-6302.

Freed, A. M. (2005), Earthquake triggering by static, dynamic, and postseismic stress transfer, Annu. Rev. Earth Planet. Sci., 33, 335-367.

Gardner, J. K., and L. Knopoff (1974), Is the sequence of earthquakes in sSouthern California, with aftershocks removed, Poissonian?, Bull. Seismol. Soc. Am., 64, 1363-1367.

Gross, S., and C. Kisslinger (1994), Estimating tectonic stress rate and state with Landers aftershocks, J. Geophys. Res., 102, 7603-7612.

Hainzl, S., A. Christophersen, and B. Enescu (2008), Impact of earthquake rupture extensions on parameter estimations of point-process models, Bull. Seismol. Soc. Am., 98(4), 2066-2072, doi:10.1785/0120070256.

Helmstetter, A. (2003), Is earthquake triggering driven by small earthquakes?, Phys. Rev. Lett., 91(5), 058501, doi:10.1103/PhysRevLett. 91.058501 .

Helmstetter, A., and B. E. Shaw (2006), Relation between stress heterogeneity and aftershock rate in the rate-and-state model, J. Geophys. Res., 111, B07304, doi:10.1029/2005JB004077.

Helmstetter, A., Y. Y. Kagan, and D. D. Jackson (2005), Importance of small earthquakes for stress transfers and earthquake triggering, J. Geophys. Res., 110, B05S08, doi:10.1029/2004JB003286.

Herrero, A., and P. Bernard (1994), A kinematic self-similar rupture process for earthquakes, Bull. Seismol. Soc. Am., 84, 1216-1288.

Kagan, Y. Y., and H. Houston (2005), Relation between mainshock rupture process and Omori's law for aftershock moment release rate, Geophys. J. Int., 163(3), 1039-1048.

Kilb, D., and J. Gomberg (1999), The initial subevent of the 1994 Northridge, California, earthquake: Is earthquake size predictable?, J. Seismol., 3, 409-420.

King, G. C. P., and M. Cocco (2001), Fault interaction by elastic stress changes: New clues from earthquake sequences, Adv. Geophys., 44, $1-$ 38 .

Kisslinger, C. (1993), The stretched exponential function as an alternative model for aftershock decay rate, J. Geophys. Res., 98, 1913-1921.

Lapusta, N., and J. R. Rice (2003), Nucleation and early seismic propagation of small and large events in a crustal earthquake model, J. Geophys. Res., 108(B4), 2205, doi:10.1029/2001JB000793.

Lavallée, D., and R. J. Archuleta (2003), Stochastic modeling of slip spatial complexities for the 1979 Imperial Valley, California, earthquake, Geophys. Res. Lett., 30(5), 1245, doi:10.1029/2002GL015839.

Lavallée, D., and R. J. Archuleta (2005), Coupling of the random properties of the source and the ground motion for the 1999 Chi-Chi earthquake, Geophys. Res. Lett., 32, L08311, doi:10.1029/2004GL022202.

Mai, P. M., and G. C. Beroza (2002), A spatial random field model to characterize complexity in earthquake slip, J. Geophys. Res., 107(B11), 2308, doi:10.1029/2001JB000588.

Marsan, D. (2006), Can coseismic stress variability suppress seismicity shadows? Insights from a rate-and-state friction model, J. Geophys. Res., 111, B06305, doi:10.1029/2005JB004060.

Marsan, D., and O. Lengliné (2008), Extending earthquakes' reach through cascading, Science, 319, 1076-1079.
Mikumo, T., and T. Miyatake (1995), Heterogeneous distribution of dynamic stress drop and relative fault strength recovered from the results of wave-form inversion-The 1984 Morgan-Hill, California, earthquake, Bull. Seismol. Soc. Am., 85, 178-193.

Miyazaki, S., P. Segall, J. Fukuda, and T. Kato (2004), Space time distribution of afterslip following the 2003 Tokachi-oki earthquake: Implications for variations in fault zone frictional properties, Geophys. Res. Lett., 31, L06623, doi:10.1029/2003GL019410.

Molchan, G. M., and O. E. Dmitrieva (1992), Aftershock identification: Methods and new approaches, Geophys. J. Int., 109, 501-516.

Montési, L. G. J. (2004), Controls of shear zone rheology and tectonic loading on postseismic creep, J. Geophys. Res., 109, B10404, doi:10.1029/2003JB002925

Narteau, C., P. Shebalin, and M. Holschneider (2002), Temporal limits of the power law aftershock decay rate, J. Geophys. Res., 107(B12), 2359 , doi:10.1029/2002JB001868.

Ouillon, G., and D. Sornette (2005), Magnitude-dependent Omori law: Theory and empirical study, J. Geophys. Res., 110, B04306, doi:10.1029/2004JB003311

Parsons, T. (2008), Persistent earthquake clusters and gaps from slip on irregular faults, Nat. Geosc., 1, 59-63.

Peng, Z., J. E. Vidale, and H. Houston (2006), Anomalous early aftershock decay rate of the 2004 Mw6.0 Parkfield, California, earthquake, Geophys. Res. Lett., 33, L17307, doi:10.1029/2006GL026744.

Peng, Z., J. E. Vidale, M. Ishii, and A. Helmstetter (2007), Seismicity rate immediately before and after main shock rupture from high-frequency waveforms in Japan, J. Geophys. Res., 112, B03306, doi:10.1029/ 2006JB004386.

Perfettini, H., and J.-P. Avouac (2004), Postseismic relaxation driven by brittle creep: A possible mechanism to reconcile geodetic measurements and the decay rate of aftershocks, application to the Chi-Chi earthquake, Taiwan, J. Geophys. Res., 109, B02304, doi:10.1029/2003JB002488.

Reasenberg, P. (1985), Second-order moment of central California seismicity, 1969-1982, J. Geophys. Res., 90, 5479-5495.

Ripperger, J., and P. M. Mai (2004), Fast computation of static stress changes on 2D faults from final slip distributions, Geophys. Res. Lett., 31, L18610, doi:10.1029/2004GL020594.

Rubin, A. M. (2002), Aftershocks of microearthquakes as probes of the mechanics of rupture, J. Geophys. Res., 107(B7), 2142, doi:10.1029/ 2001JB000496.

Rubin, A. M., and J.-P. Ampuero (2005), Earthquake nucleation on (aging) rate and state faults, J. Geophys. Res., 110, B11312, doi:10.1029/ 2005JB003686.

Schmittbuhl, J., G. Chambon, A. Hansen, and M. Bouchon (2006), Are stress distributions along faults the signature of asperity squeeze?, Geophys. Res. Lett., 33, L13307, doi:10.1029/2006GL025952.

Sornette, D., and G. Ouillon (2005), Multifractal scaling of thermally activated rupture processes, Phys. Rev. Lett., 94(3), 038501, doi:10.1103/ PhysRevLett.94.038501.

Steacy, S., J. Gomberg, and M. Cocco (Eds.) (2005), Special section on Stress Transfer, Earthquake Triggering, and Time-Dependent Hazard, J. Geophys. Res., 110.

Turcotte, D. L. (1997), Fractals and Chaos in Geology and Geophysics, Cambridge Univ. Press, Cambridge, U.K

Utsu, T., Y. Ogata, and R. S. Matsu'ura (1995), The centenary of the Omori formula for a decay of aftershock activity, J. Phys. Earth, 43, 1-33.

Wells, D. L., and K. J. Coppersmith (1994), New empirical relationships among magnitude, rupture length, rupture width, rupture area, and surface displacement, Bull. Seismol. Soc. Am., 84, 974-1002.

Zhang, W., T. Iwata, K. Irikura, H. Sekiguchi, and M. Bouchon (2003), Heterogeneous distribution of the dynamic source parameters of the 1999 Chi-Chi, Taiwan, earthquake, J. Geophys. Res., 108(B5), 2232, doi:10.1029/2002JB001889.

Zhuang, J., Y. Ogata, and D. Vere-Jones (2004), Analyzing earthquake clustering features by using stochastic reconstruction, J. Geophys. Res., 109, B05301, doi:10.1029/2003JB002879.

Zhuang, J., C.-P. Chang, Y. Ogata, and Y.-I. Chen (2005), A study on the background and clustering seismicity in the Taiwan region by using point process models, J. Geophys. Res., 110, B05S18, doi:10.1029/ 2004JB003157.

S. Hainzl, Helmholtz-Zentrum Potsdam: Deutsches GeoForschungsZentrum, Section 2.1, Telegrafenberg, 14473 Potsdam, Germany. (hainzl@gfz-potsdam.de)

D. Marsan, Laboratoire de Geophysique Interne et Tectonophysique, Universite de Savoie, F-73376 Le Bourget du Lac, France. (david.marsan@ univ-savoie.fr) 